\title{
The Performance of Energy Dispersive X-ray Analysis at high Temperatures using a windowless Multi Detector XEDS System
}

\author{
Christoph Mitterbauer ${ }^{1}$, Mikhail Ovsyanko ${ }^{1}$, Yuri Rikers ${ }^{1}$, Bert Freitag $^{1}$ \\ ${ }^{1 .}$ Thermo Fisher Scientific, Achtseweg Noord 5, Eindhoven, The Netherlands
}

Energy dispersive $\mathrm{x}$-ray analysis (XEDS) has increased its sensitivity with the introduction of windowless multi detector systems in transmission electron microscopy. Today routinely atomic resolution compositional maps and maps with big field of view (512x512 pixel) can be acquired within minutes of acquisition time[1]. Here the increased sensitivity due to the higher collection angles $(\sim 1$ $2 \mathrm{srad})$, the use of SDD detectors with low deadtime and the windowless design are the main reason for this achievement[2]. Nevertheless windowless design creates a problem in detection at elevated temperatures since the sensors see the light of the heater element of the holder, which flood the sensor with signal and makes work at above $400{ }^{\circ} \mathrm{C}$ impossible [3]. Even the use of MEMS based holders[4] with reduced heated area does not prevent this problem in XEDS analysis at high temperatures. The traditional way of preventing this effect is to introduce a metal window in front of the sensor to block the light of the heater to reach the sensor. But this approach reduces the sensitivity of the system significantly as you can see in the graph in figure 1, which compares windowless with windowed SDD detector in sensitivity. This strong decline in sensitivity is already caused by a thin polymer window, which still allows part of the light of the heater to reach the sensor and the effect using a metal window (Be,Al) due to its higher mass thickness is even more dramatic and not optimum for heating experiments using XEDS.

In this contribution we present data of 2 windowless XEDS systems (DualX and SuperX) in XEDS analysis up to $750{ }^{\circ} \mathrm{C}$ using a MEMS based heating holder. The performance of the systems is benchmarked in dead time, energy resolution and sensitivity in a temperature range from room temperature up to $750^{\circ} \mathrm{C}$ (figure 2). The benefits of having higher sensitivity especially in the low energy range compared to a windowed sensor are illustrated. The newly introduced SuperX G2 electronics allows to acquire XEDS spectra and maps up to $750^{\circ} \mathrm{C}$ with the windowless four sensor SuperX detectors on Talos or Themis platforms. A comparison between a large 2 detector XEDS system (DualX) with conventional electronics and the 4 detector XEDS system (SuperX) in its behaviour at different sample temperature is discussed. We demonstrate in multiple examples that with the redesign of the SuperX electronics XEDS analysis with high sensitivity over the entire energy range can be performed up to $750^{\circ} \mathrm{C}$ without sensitivity loss caused by a windowed design.

\section{References:}

[1] LJ Allen et al, MRS Bulletin 37 (2012), p. 47

[2] P Schlossmacher et.al, , Microscopy Today 18(04), (2010), p. 14

[3] I. Ohnishi et al, MC Lausanne proceedings (2017) p. 584

[4] L Mele et al, Microscopy Research and Technique 79 (2016) p. 239 


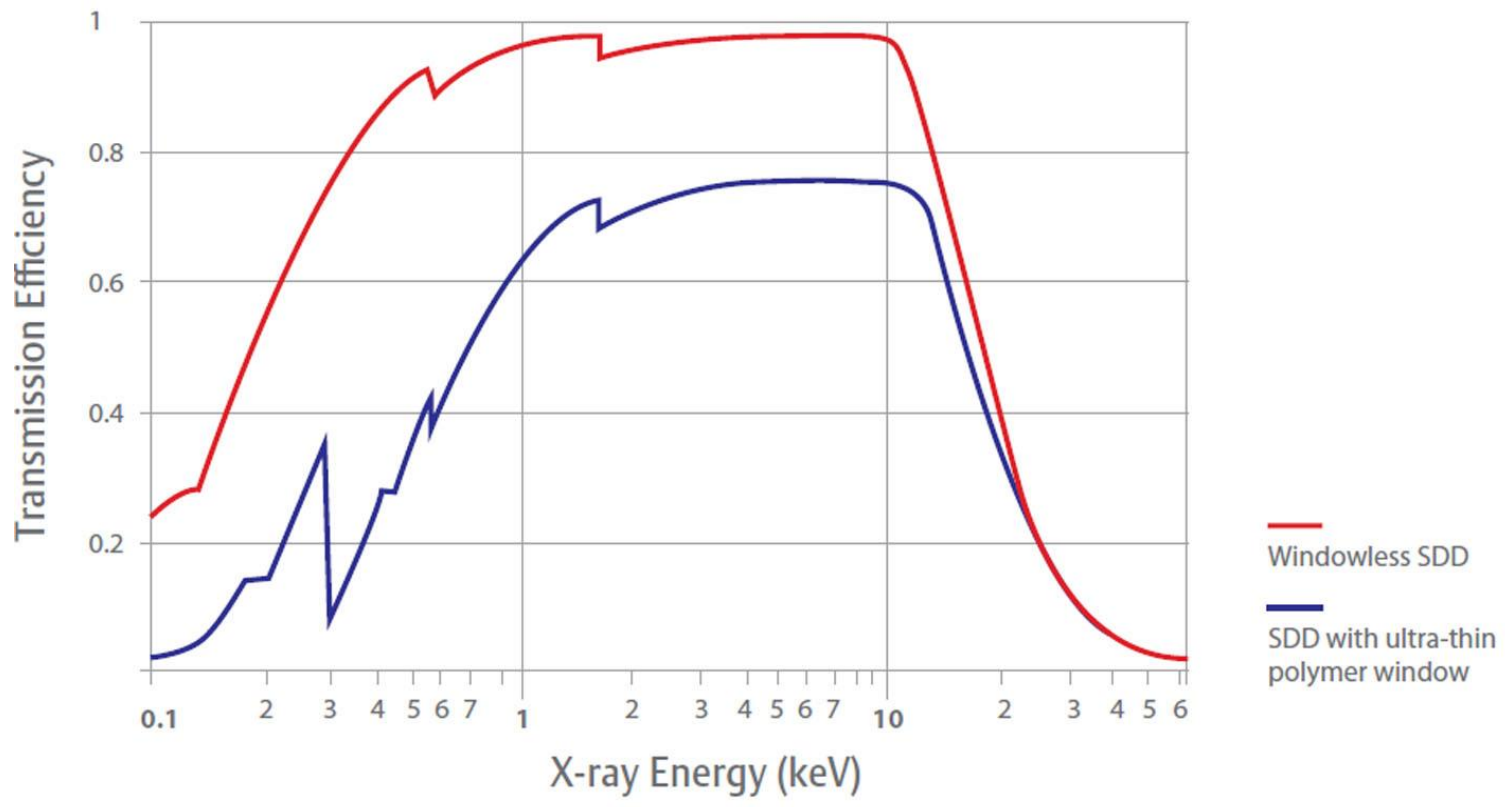

Figure 1. X-ray transmission efficiency versus energy for a windowless SDD detector (red curve) and an SDD detector with thin polymer window (blue curve). Loss due to the both the Si grid bars and the polymer window contributes to the lower efficiency across all energies of the detector with window. the dramatic loss of signal not only at low $(<2 \mathrm{keV})$ but as well relatively high energies $(\sim 10 \mathrm{keV})$ is visible.

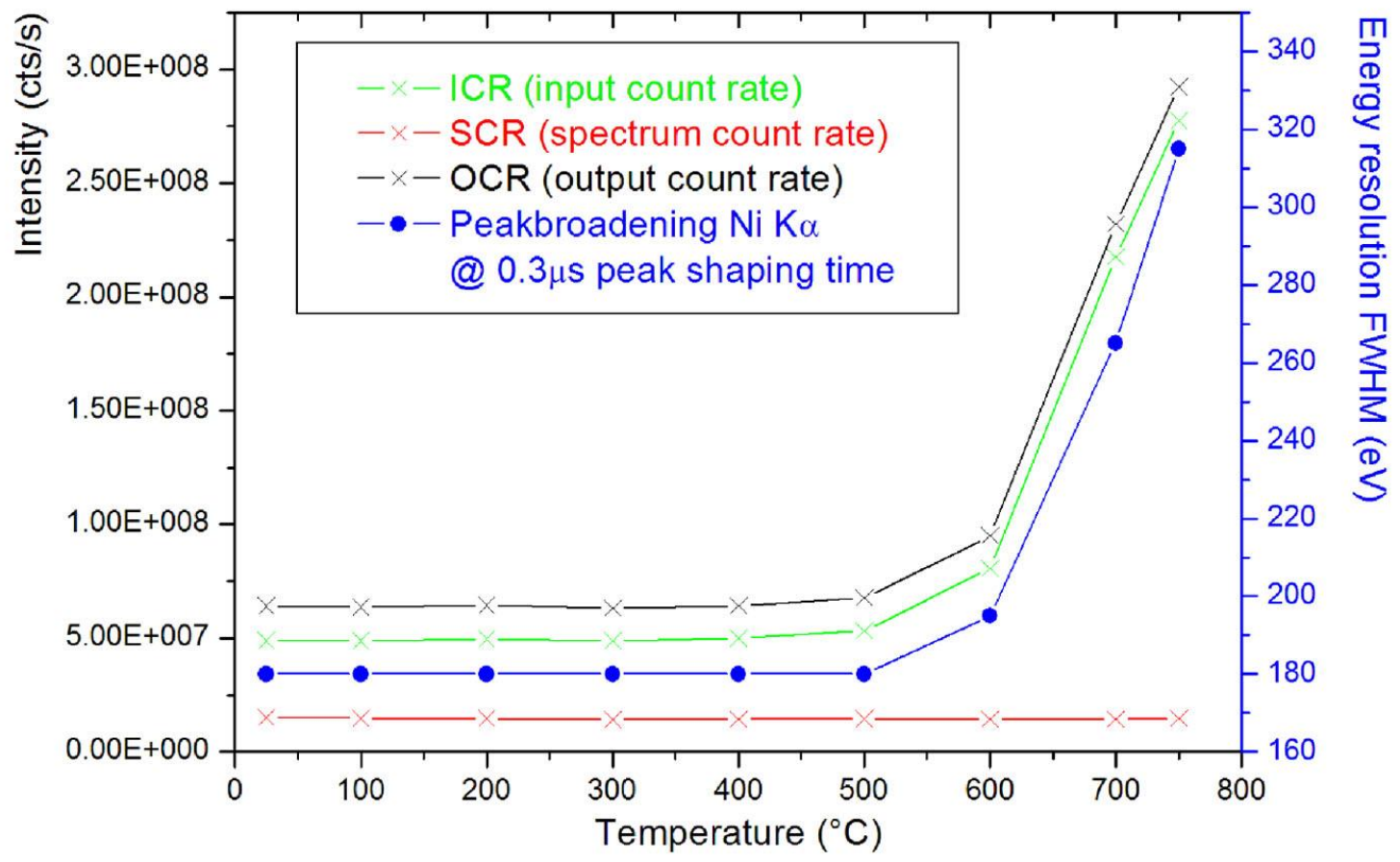

Figure 2. Measurement of the input count rate, spectrum count rate and output count rate and energy resolution on Nickel K alpha line of the SuperX G2 detector with new electronic in dependence of temperature using a MEMS heating holder on a Talos platform. The temperature influences the energy resolution, but does not change the count rate in the spectrum significantly. 